\title{
Alianza del Pacífico y Mercado Común del Sur: dos enfoques de la integración económica regional*
}

\author{
The Pacific Alliance and Mercosur: Two \\ Approaches to Economic Regional Integration
}

\author{
Alberto Martínez Castillo**
}

Recibido: 6 de octubre de 2016

Aprobado 1 de noviembre de 2016

Disponible en línea: 20 de diciembre de 2016

\section{Resumen}

Tanto el Mercado Común del Sur (Mercosur) como la Alianza del Pacífico (AP) son acuerdos de integración económica regional, cuyos principios constitutivos se ajustan al denominado regionalismo abierto. Sin embargo, cuando nace la AP, con la Declaración de Lima del 28 de abril de 2011, el Mercosur estaba alejado del liberalismo económico, y su agenda política, que había adquirido especial relevancia, lo condujo a enfrentamientos con los Estados Unidos. Algunos analistas llegaron a hablar de una confrontación entre los librecambistas eficientes de la AP y los estatistas ineficientes del Mercosur. En este artículo, se hace un análisis de ambos organismos regionales. La investigación, basada en fuentes documentales y estadísticas, pretende evaluar la capacidad de estos organismos para impulsar el desarrollo económico mediante la integración regional, objetivo propuesto en sus documentos constitutivos. La principal conclusión es que la

\section{Abstract}

Both the Pacific Alliance (PA) and the Common Market of the South (Mercosur) are economic regional integration agreements whose constitutive principles conform to the so-called open regionalism. However, when the AP was launched in 2011, with the Declaration of Lima, Mercosur had distanced from economic liberalism, and its political agenda, which had acquired especial relevance, led it to clashes with the United States. Some analysts came to speak of a confrontation between the efficient liberalizers of the PA and the inefficient statists of Mercosur. In this paper, I analyze both regional agreements. The research, based on documentary sources and statistics, aims to evaluate the ability of these two bodies to promote economic development through regional integration, an objective proposed in its constituent documents. The main conclusion is that AP is better prepared to achieve the proposed objective because of its

doi:10.11144/Javeriana.papo21-2.apmc

* Artículo de reflexión.

** Universidad Simón Bolívar (Caracas, Venezuela). Correo electrónico: amartine@usb.ve 
AP está mejor preparada para lograr el objetivo propuesto debido a su compromiso con la integración profunda y el comercio de cadenas globales de valor. Otra es que la política y el enfrentamiento ideológico se han convertido en un elemento perturbador del desempeño del Mercosur.

\section{Palabras clave}

Alianza del Pacífico; Mercosur; integración regional; integración económica; cadenas globales de valor

\section{Cómo citar este artículo:} Martínez-Castillo, A. (2016). Alianza del Pacífico y Mercado Común del Sur: dos enfoques de la integración económica regional. Papel Político, 21(2), 469-494. https://doi.org/10.11144/ Javeriana.papo21-2.apmc commitment to deep integration and trade of global value chains. Another concluding remark is that political and ideological confrontation have become a disturbing element in the performance of Mercosur.

\section{Keywords}

Pacific Alliance; Mercosur; regional integration; economic integration; global value chains 


\section{Introducción}

El término integración regional ha sido definido de diferentes maneras, lo cual ha generado a veces confusión. El origen del problema estriba en que el término se ha usado para definir tanto una condición final como el proceso para llegar a esa condición final (Heinonen, 2006). Aquellos que destacan la parte procedimental del fenómeno consideran la integración regional como un proceso mediante el cual unos países deciden cooperar entre sí para alcanzar objetivos comunes que da como resultado un mayor acercamiento entre ellos. Dicha cooperación puede ser en diversos campos, tales como el político, el económico, el militar o el ambiental. El problema con este enfoque estriba en que no define cuál es ese estado de mayor acercamiento (condición final) o en qué momento se logra.

Los que enfocan la integración desde el punto de vista de la condición final señalan hasta dónde debe llevar el proceso. Por ejemplo, en el caso de la integración política, los federalistas afirman que la integración regional debe llevar a la formación de una unión federal entre los Estados miembros, es decir, una nueva comunidad política que se impone sobre las que ya existían. Si de integración económica se trata, un ejemplo podría ser el establecimiento de una unión monetaria.

La Comisión Europea define la integración regional como

the process of overcoming barriers that divide neighboring countries, by common accord, and of jointly managing shared resources and assets. Essentially, it is a process by which groups of countries liberalize trade, creating a common market for goods, people, capital and services. The European Union advocates regional integration as an effective means of achieving prosperity, peace and security. ${ }^{1}$ (European Commission, s. f.)

Es decir, la integración está definida como proceso de liberación del comercio y como condición final el mercado común.

Por su parte, la integración regional se fundamenta en tres pilares: 1) el comercio, 2) la inversión extranjera y 3) una gama de asuntos políticos, sociales y técnicos (Asian Development Bank, Asian Development Bank Institute y Inter-American Development Bank, 2012). Usualmente, el comercio guía el proceso y genera suficiente información para que ocurra la inversión extranjera. Cuando hay una masa crítica de comercio e inversión, aumentan los incentivos para que los Gobiernos cooperen en asuntos políticos, sociales y técnicos.

Como se puede apreciar, ambos organismos ponen el acento en la integración económica que, de ser exitosa, conduce a la integración política. Cabe señalar que, en

\footnotetext{
${ }^{1}$ El proceso de superar las barreras que dividen a los países vecinos y de manejar activos y recursos compartidos de manera mancomunada. Esencialmente, es un proceso mediante el cual grupos de países liberalizan el comercio y crean un mercado común de bienes, gente, capital y servicios. La Unión Europea promueve la integración regional como un medio efectivo de alcanzar prosperidad, paz y seguridad.
} 
los procesos de integración regional, hay interdependencia entre los factores económicos y políticos, de tal modo que la integración económica puede, eventualmente, motivar la integración política o, por el contrario, los factores políticos conducirían a la integración económica (Balassa, 2014).

Los primeros acuerdos de integración regional latinoamericanos, tales como la Asociación Latinoamericana de Libre Comercio, el Mercado Común Centroamericano y la Comunidad Andina (CAN), ${ }^{2}$ ocurrieron en la década de 1960 y tenían como objetivo principal incrementar el comercio entre los países miembros para promover la industrialización y el crecimiento económico. Para ello, se pretendía eliminar los aranceles al comercio intrarregional manteniendo la protección arancelaria para las importaciones provenientes de países no miembros, estrategia que Gil y Paikin (2013) denominaron regionalización hacia dentro, y otros autores regionalismo cerrado o desarrollista. Sin embargo, estos intentos de integración fueron poco exitosos debido, principalmente, al alto proteccionismo propiciado por la política de industrialización por sustitución de importaciones y el clima negativo que existía para la inversión extranjera (Martínez, 2015).

En la década de 1980, ocurrieron dos eventos que impactaron la estrategia de integración en la región. Uno fue la firma del Acta Única Europea, por parte de los miembros de la Unión Europea, para permitir la libre circulación de bienes, servicios, capital y personas. Esta profundización de la integración europea inició un debate sobre los efectos de la integración económica en el crecimiento de las economías nacionales y su papel en la promoción de la productividad y la competitividad.

El otro evento fue la crisis de la deuda externa latinoamericana que puso en evidencia el agotamiento de la industrialización por sustitución de importaciones. ${ }^{3}$ Los países latinoamericanos respondieron con programas de ajuste macroeconómico que incluían la liberalización del comercio internacional y la reducción del tamaño del Estado para dar un mayor papel al mercado en la asignación de los recursos productivos. La reducción unilateral de aranceles facilitó la integración regional, la hizo menos costosa y permitió el nacimiento del denominado regionalismo abierto, caracterizado por bajos niveles de protección contra la competencia externa, con el fin de incentivar la eficiencia y atraer inversión extranjera. Según la Comisión Económica para América Latina y el Caribe (Cepal), el regionalismo abierto buscaba que las políticas de integración fueran compatibles y complementarias con políticas diseñadas para mejorar la competitividad internacional (Cepal, 1994). En 1989, la CAN adopta el regionalismo abierto y le da un fuerte impulso al hasta entonces estancado comercio intrarregional (Martínez, 2015).

\footnotetext{
${ }^{2}$ La CAN se creó en 1969 con el nombre de Pacto Andino.

${ }^{3}$ La protección del mercado local eliminó el incentivo para que las empresas fueran eficientes y produjeran para los mercados externos, lo cual terminó generando presiones sobre la cuenta corriente de la balanza de pagos.
} 
Dos años más tarde nace el Mercado Común del Sur (Mercosur) y en corto tiempo se proyecta como ejemplo exitoso de regionalismo abierto.

También en la década de 1980 ocurren cambios importantes en el ámbito de la política internacional. En 1983, Colombia y México proponen a Venezuela y Panamá crear una instancia multilateral, que recibió el nombre de Grupo de Contadora, con el objeto de mediar en el restablecimiento de la paz en Centroamérica, afectada por conflictos armados en El Salvador, Guatemala y Nicaragua. El Grupo no contó con el apoyo de los Estados Unidos que no reconocían el Gobierno de Nicaragua, pero sí con el apoyo de Argentina, Brasil, Perú y Uruguay, países que, en 1985, conformaron el Grupo de Contadora. Posteriormente, los ocho países ampliaron sus preocupaciones a otros temas de interés regional y en 1990 formaron el Grupo de Río, mecanismo de consulta y concertación política que veinte años más tarde se transformaría en la Comunidad de Estados Latinoamericanos y Caribeños y que incorporaría a treinta y tres países de la región, con el fin de promover la integración y el desarrollo. Estas experiencias de coordinación política regional sentaron las bases para incluir en los acuerdos de integración objetivos políticos (Gil y Paikin, 2013; Sanahuja, 2007).

Los programas de ajuste macroeconómico tuvieron un fuerte impacto sobre los grupos de menores ingresos. Entre 1990 y 2005, el coeficiente de Gini pasó de 0.509 a 0.512 en América Latina. ${ }^{4}$ La lucha contra la pobreza fue una bandera de los partidos de izquierda que empezaron a cosechar victorias electorales con Hugo Chávez en 1999, Néstor Kirchner y Luiz Inácio Lula da Silva en 2003, y Tabaré Vázquez y Evo Morales en 2005..$^{5}$ Estos gobernantes se manifestaron críticos de la capacidad de los mercados para promover el crecimiento económico y la superación de la pobreza y, apoyados en los ingresos generados por el incremento en los precios internacionales de las materias primas, favorecieron la intervención del Estado en la actividad económica. Además, demandaron mayor autonomía en su relación con los Estados Unidos (Goulart, 2016).

En el contexto descrito, nació lo que algunos autores han denominado regionalismo posliberal (Sanahuja, 2012) o regionalismo poshegemónico (Riggirozzi y Tussie, 2012), caracterizado por un retorno a la política y menor atención a la economía, oposición al neoliberalismo y a la hegemonía de los Estados Unidos, desconfianza en los mercados, aumento del intervencionismo estatal y creciente atención a temas sociales, como la pobreza, la desigualdad y la inclusión.

\footnotetext{
${ }^{4}$ El incremento de la desigualdad del ingreso es un hecho generalizado. Por ejemplo, en tres cuartas partes de los países de la Organización para la Cooperación y el Desarrollo Económico (OCDE), los ingresos de $10 \%$ de los hogares más ricos crecieron más rápido que los ingresos de $10 \%$ de los hogares más pobres durante los veinte años que precedieron a la crisis financiera de 2008 (Cingano, 2014). Picketty (2014) afirma que la desigualdad del ingreso ha aumentado en las últimas décadas, porque la tasa de rendimiento del capital ha sido mayor a la tasa de crecimiento del ingreso.

${ }^{5}$ Posteriormente, la izquierda ganó las elecciones presidenciales en Ecuador (2006), Paraguay (2008) y Perú (2011).
} 
Paralelamente, surge otro regionalismo donde la economía desempeña el papel principal: el regionalismo del siglo XXI. De acuerdo con Baldwin (2011), la reducción en los costos de transporte y el desarrollo de las tecnologías de la información y de la comunicación permitieron fragmentar el proceso de producción y llevarlo a cabo en diferentes países, lo cual dio origen a las redes de producción internacional y al comercio de cadenas globales de valor (CGV). Dicho comercio se produce cuando las empresas transnacionales de alta tecnología combinan su know-how con mano de obra barata de los países en desarrollo adonde mudan algunas etapas del proceso de producción. Se crea entonces un flujo de bienes, servicios, gente e ideas y el nexo comercio-inversiónservicios que responde a dos necesidades de las empresas multinacionales: conectar fábricas y hacer negocios en el exterior.

Las CGV exigían ir más allá de la simple reducción de aranceles, porque las empresas multinacionales requerían un marco regulatorio adecuado que protegiera la inversión extranjera, los derechos de propiedad y facilitara hacer negocios. Los países en desarrollo deseosos de atraer fábricas de tecnología avanzada se mostraron dispuestos a suscribir acuerdos de integración profunda para regular estos temas, lo cual dio origen al regionalismo del siglo XXI (Baldwin, 2011).

En el presente artículo, se estudian dos acuerdos de integración regional: el Mercosury la Alianza del Pacífico (AP). El objetivo principal es explorar la posibilidad que tienen estos dos organismos de impulsar el crecimiento económico. Apoyado en fuentes documentales y estadísticas, el análisis combina elementos cualitativos y cuantitativos en su diseño de investigación. En la sección uno, se analiza el Mercosur, en la dos la AP, la tres trata el tema de las instituciones y el desempeño económico, en la cuatro se discute la posible convergencia entre los dos acuerdos y, finalmente, se presentan las conclusiones. La principal conclusión es que la AP está mejor preparada para lograr el objetivo propuesto debido a su compromiso con la integración profunda y el comercio de cadenas globales de valor.

\section{Mercosur: del regionalismo abierto al regionalismo posliberal}

El 26 de junio de 1991 Argentina, Brasil, Uruguay y Paraguay firmaron el Tratado de Asunción, con el objetivo de acelerar el desarrollo económico mediante la creación del Mercosur. Para ello, se comprometían a los siguiente: 1) permitir la libre circulación de bienes, servicios y factores productivos, 2) adoptar un arancel externo común (AEC) y una política comercial común con relación a terceros Estados, 3) coordinar las políticas macroeconómicas y 4) armonizar las legislaciones para fortalecer el proceso de integración. El Mercosur nació en un contexto de ajuste estructural y liberación económica y adoptó el regionalismo abierto promovido por la Cepal.

En sus inicios, el Mercosur tuvo efectos positivos sobre el comercio intrazona, que de 8 \% en 1991 pasó a 25 \% en 1998, su punto más elevado (Martínez, 2015). Sin embargo, 
los choques externos, como los de la crisis asiática de finales de la década de 1990 y la crisis financiera de 2008, pusieron en evidencia la incapacidad de los Estados partes para coordinar sus políticas macroeconómicas. Por el contrario, cada uno actuó unilateralmente protegiendo los intereses nacionales e incumpliendo el compromiso regional de libre circulación de bienes y servicios. Mientras Brasil recurrió, principalmente, a ajustes del tipo de cambio, Argentina prefirió la imposición de barreras administrativas al comercio, tales como derechos anti-dumping y licencias no automáticas de importación, con lo cual se convirtió en el Estado parte con más trabas al comercio intrarregional. Esta dinámica condujo a “un 'nuevo proteccionismo' para 'evitar contagios' a partir del comercio, y preservar los mercados nacionales desde una óptica mercantilista" (Zelicovitch, 2015, p. 103); es decir, se privilegiaron los mercados nacionales a expensas del mercado regional. Después de China, Brasil es el segundo país al que Argentina le aplica más medidas anti-dumping. El resultado fue la caída del comercio intrazona hasta ubicarse en 13.6 \% para 2015, cifra baja comparada con las registradas por el Tratado de Libre Comercio de América del Norte y la Unión Europea, que superan 50 y $60 \%$, respectivamente.

En cuanto al AEC, ha sido objeto de modificaciones y perforaciones cada vez más frecuentes. Mientras en 1999 la Comisión de Comercio del Mercosur dedicó 20 \% de sus tareas a tratar casos de excepciones al AEC, en 2014 el porcentaje aumentó a 60 \% (Zelicovitch, 2015). Y la posibilidad de alcanzar la unión aduanera se alejó en la cumbre de Brasilia celebrada en julio de 2015, cuando se decidió prorrogar por varios años las excepciones al AEC y la aplicación de regímenes especiales de comercio (Banco Interamericano de Desarrollo [BID], 2015). De modo que las tendencias proteccionistas siguen privilegiando lo intereses nacionales a expensas de los intereses comunitarios.

Sobre política comercial común también falta mucho por hacer, como lo ponen en evidencia los cuatro ejemplos citados a continuación. Uno, en la Organización Mundial del Comercio (OMC), los miembros del Mercosur formalmente actúan de manera individual, y en las negociaciones de la Ronda de Doha de 2008, Brasil actuó en contra de lo acordado previamente por el bloque (Zelicovitch, 2015), tras lo cual se mostró dispuesto a aceptar, junto con Uruguay y Paraguay, la propuesta del entonces director general de la OMC, Pascal Lamy, sobre reducción de aranceles a los bienes industriales, pero Argentina se opuso. Dos, en la negociación con la Unión Europea para establecer una zona de libre comercio entre los dos bloques, iniciadas en 2000, suspendidas en 2004 y retomadas en 2010, aún no se ha logrado un acuerdo, en parte por la oposición de Argentina y sus desacuerdos con Brasil, Paraguay y Uruguay, países que consideran la política comercial argentina excesivamente proteccionista (Makuc, Duhalde y Rozemberg, 2015). Tres, en la renegociación del Acuerdo de Complementación Económica número 55, que regula el intercambio automotor entre México y el Mercosur, propiciado 
por Argentina y Brasil para prorrogar la entrada en vigor del libre comercio hasta 2019, “primó el interés específico de cada Estado parte, sin mediar ningún tipo de coordinación ni articulación previa entre las autoridades de Argentina y Brasil” (BID, 2015, p. 102), a pesar de que ambos países habían acordado unir esfuerzos en la negociación con México. Cuatro, el Mercosur no tiene una estrategia colectiva para relacionarse con China, país que ha aumentado su presencia en la región (Goulart, 2016). La falta de coordinación sobre política comercial externa y el excesivo proteccionismo son dos elementos que ayudan a explicar por qué el grupo apenas ha firmado dos acuerdos de libre comercio con Israel y la Unión Aduanera Sudafricana, socios comerciales de poco peso para el grupo.

Respecto del objetivo de armonizar las legislaciones de los Estados partes, el progreso ha sido lento. Un informe de la Secretaría del Mercosur de 2004 estimó que 67 \% de las normas comunitarias no habían sido internalizadas ${ }^{6}$ en los ordenamientos jurídicos nacionales (BID, 2009), razón por la cual se decidió que las normas no internalizadas por ninguno de los Estados partes pasados cinco años desde su aprobación deben ser nuevamente analizadas por el órgano decisorio del cual emanaron. El atraso en la internalización puede explicarse por lo largo del proceso, que consta de tres pasos. Primero, los Estados partes adoptan las medidas necesarias para la incorporación de la norma aprobada al ordenamiento jurídico nacional y, una vez hecho esto, lo informan a la Secretaría Administrativa del Mercosur. Cuando la Secretaría tiene la información de todos los Estados partes, procede a comunicarlo a cada uno de ellos, cumpliendo con el segundo paso. El tercer paso se produce treinta días después de la comunicación de la Secretaría, cuando la norma entra en vigor en todos los Estados partes. Otra razón es que cada Estado decide su propia manera de internalizar la norma y el tiempo que le tomará.

El problema de la internalización podría resolverse si los Estados partes decidieran darles aplicabilidad inmediata a las normas comunitarias, para lo cual tendrían que crear instituciones supranacionales que le den carácter supranacional al sistema jurídico del Mercosur. Bergamaschine (2005, p. 243) afirma: “El proceso de integración, al implementarse la supranacionalidad integral, podría dar el salto definitivo hacia el mercado común, favoreciendo la creación de una mayor seguridad jurídica y económica de la región, y promoviendo un mayor desarrollo para los miembros y asociados".

La renuencia de los Estados partes a crear instituciones supranacionales ha conferido un poder importante a sus presidentes a la hora de fijar el rumbo del Mercosur, lo cual afecta la estabilidad institucional del organismo. Por ejemplo, a mediados de la primera década del presente siglo, los presidentes Lula da Silva, Néstor Kirchner y Tabaré Vásquez, junto con Hugo Chávez, cuyo país ingresó como Estado asociado en

\footnotetext{
${ }^{6}$ La internalización se refiere a la adopción y aplicación, en el derecho de cada país, de las decisiones del Consejo de Mercado Común, las resoluciones del Grupo de Mercado Común y las directivas de la Comisión de Comercio. La Secretaría de Mercosur no ha vuelto a publicar cifras de internalización.
} 
2004, alejaron al Mercosur del regionalismo abierto y le dieron prioridad a lo político sobre lo económico e introdujeron en la agenda temas sociales, como la pobreza o la desigualdad del ingreso, lo cual terminó dándole un perfil de regionalismo posliberal al organismo. Ese poder se manifestó nuevamente en junio de 2012 cuando Dilma Rousseff, Cristina Fernández y José Mujica decidieron suspender a Paraguay, cuyo parlamento se negaba a ratificar el protocolo de adhesión de Venezuela, ${ }^{7}$ e inmediatamente admitieron a este último país como miembro pleno.

Es claro que la introducción de temas sociales fortaleció al Mercosur al abrir las puertas para implementar políticas públicas en las áreas de educación y salud, necesarias para que los efectos positivos de la integración lleguen a la población de menores ingresos. Sin esas políticas, la integración podría beneficiar solo a unos pocos (Tugores, 2006).

En cuanto a los temas políticos, estos han estado presentes desde los orígenes del Mercosur. No hay que olvidar que Brasil y Argentina inician el proceso de integración con la firma del Acta de Foz de Iguazú para dejar atrás "el paradigma del conflicto y de la puja de liderazgos entre los grandes países de América del Sur” (Caetano, 2011, p. 31). Sin embargo, darle prioridad a lo político y poner lo económico en segundo plano alejó al Mercosur de los objetivos propuestos en el Tratado de Asunción. Además, permitió introducir elementos ideológicos que pretendían utilizar el organismo regional como una plataforma para enfrentar la hegemonía de los Estados Unidos en la región, criticar sus políticas neoliberales y atacar a países latinoamericanos ajenos a la ideología de izquierda. Ese fue el objetivo principal de Chávez cuando Venezuela ingresó al organismo regional (Martínez, 2015). Distanciado de la CAN, que calificaba como proyecto neoliberal basado en el comercio, Chávez consiguió que la Cumbre Presidencial del Mercosur aprobara la membrecía de Venezuela en diciembre de 2005. El protocolo de adhesión plena se firmó en julio de 2006 y fue ratificado ese mismo año por los parlamentos de Argentina y Uruguay, controlados por partidos de izquierda, pero los de Brasil y Paraguay, donde dominaba la centroderecha, no lo ratificaron alegando el carácter poco democrático del Gobierno venezolano. Tres años más tarde Brasil lo ratifica, pero no así Paraguay. Hubo que esperar tres años adicionales y la suspensión de Paraguay para que Venezuela se convirtiera en miembro pleno. Chávez calificó como una derrota al imperialismo la suspensión de Paraguay; el presidente de este país, Federico Franco, como un acto ilegal; y José Mujica, presidente de Uruguay, como la victoria de lo político sobre lo jurídico (Martínez, 2015). La división interna en el Mercosur echaba raíces.

El enfrentamiento entre los Estados partes se agravó por el giro hacia la derecha que empezó a dar el Mercosur a partir del triunfo de Mauricio Macri en las elecciones argentinas de noviembre de 2015. En su primera rueda de prensa como presidente

${ }^{7}$ El Congreso paraguayo albergaba dudas sobre el compromiso democrático del Gobierno venezolano. 
electo, Macri manifestó que solicitaría la aplicación de la cláusula democrática ${ }^{8}$ contra Venezuela, porque el Gobierno de Nicolás Maduro perseguía a los políticos opositores y coartaba la libertad de expresión. Posteriormente, en mayo de 2016, Dilma Rousseff es apartada del cargo y tres meses más tarde destituida, pasando la presidencia de Brasil a manos de Michel Temer, militante del centrista Partido Movimiento Democrático Brasileño. El presidente Maduro calificó la destitución de Rousseff como golpe de Estado, llamó a Temer usurpador y anunció el retiro del embajador de Venezuela. La mesa estaba servida para un choque de ideologías.

En mayo de 2016, la tensión aumenta cuando Paraguay le pide a Uruguay, país que sustentaba la presidencia pro tempore, una reunión de cancilleres para analizar la situación política y social de Venezuela, iniciativa avalada por Argentina. En junio, Uruguay decide suspender la cumbre presidencial programada para julio, cuando se traspasaría la presidencia a Venezuela, porque Argentina, Brasil y Paraguay se resistían al traspaso. Según declaraciones del canciller paraguayo, Eladio Loizaga, no querían que la presidencia del Mercosur

se utilice [...] para proyectar políticas internas y generar situaciones de conflicto con otros bloques como por ejemplo la Alianza del Pacífico, con la cual el Mercosur está teniendo un diálogo más fluido y buscando un punto de convergencia cuanto antes posible [...] se instrumente hacia otros objetivos y menos la generación de conflictos por cuestiones ideológicas o dogmáticas. (Noticia al día, 2016)

El presidente uruguayo, Tabaré Vázquez, quien junto con Maduro conformaba el reducto de la izquierda en el Mercosur, insistió en que a Venezuela le correspondía asumir la presidencia. Al no haber acuerdo, su cancillería notificó que el 30 de julio Uruguay terminaría sus funciones en la presidencia. Ese mismo día Delcy Rodríguez, canciller de Venezuela, comunicó que su país asumía la presidencia del Mercosur.

Loizaga reaccionó calificando la decisión de Venezuela como una

"autoproclamación", "unilateral” y de "facto", sin validez porque el traspaso de la presidencia debía hacerse por consenso y en el marco de la Cumbre de jefes de Estado del Mercosur. Por su parte, el canciller de Brasil, José Serra, afirmó que "Venezuela vive bajo un régimen autoritario, no democrático [...] un país que tiene presos políticos no puede ser un país democrático [...] Venezuela no va a asumir el Mercosur, eso es seguro. (Angarita, 2016)

\footnotetext{
${ }^{8}$ La aplicación de la cláusula democrática se refiere a la aplicación del Protocolo de Ushuaia que establece que, de haber ruptura del orden democrático en un Estado parte, este puede ser suspendido por decisión unánime de los otros Estados partes.
} 
La posición de ambos cancilleres fue respaldada por Macri, quien señaló: "Lo que allí [en Venezuela] sucede es inaceptable. Es la violación absoluta de los derechos humanos. Hay presos políticos, existe desabastecimiento, eso es algo con lo que no se puede convivir". Y refiriéndose al Mercosur: "Creo que fortalecer el Mercosur supone tener como prioridad converger con la Alianza del Pacífico" (El Nacional, 2016). Maduro, por su parte, manifestó que Venezuela ejercería plenamente la presidencia del Mercosur pese a la persecución de la "triple alianza de torturadores de Suramérica". "Nos persigue la oligarquía paraguaya corrupta y narcotraficante, ahora nos persigue el demacrado Macri de Argentina, fracasado, repudiado por su pueblo, ahora nos persigue la dictadura impuesta por Brasil” (EFE, 2016).

Los cuatro miembros fundadores decidieron ejercer la presidencia del organismo regional de manera conjunta alegando que Venezuela tenía vencido el plazo de cuatro años que le habían dado para internalizar la normativa del Mercosur. Posteriormente, el 1 de diciembre de 2016, suspendieron la membrecía de Venezuela por no culminar el proceso de internalización. Aunque el artículo 3 del Protocolo de Adhesión de Venezuela al Mercosur establece que el país debe adoptar el acervo normativo "en forma gradual, a más tardar en cuatro años" después de la entrada en vigencia del instrumento, no prevé que la falta de internalización supone suspensión. Nuevamente, se impuso lo político sobre lo jurídico.

El enfrentamiento tomó un nuevo cariz el 2 de abril de 2017, cuando los miembros fundadores amenazaron con expulsar a Venezuela del organismo invocando la cláusula democrática. La medida fue en respuesta a la decisión del Tribunal Supremo de Justicia venezolano de despojar de sus funciones la Asamblea Nacional. La canciller de Argentina, Susana Malcorra, afirmó que el Gobierno venezolano debía “adoptar inmediatamente medidas concretas, concertadas con la oposición, para asegurar la efectiva separación de poderes, el respeto del Estado de Derecho, los derechos humanos y el respeto de las instituciones" (Rivas, 2017).

El uso del Mercosur como herramienta para la lucha política e ideológica introdujo elementos de perturbación que terminaron debilitando la institucionalidad del organismo y enfrentando a los Estados partes. Cuando prevalecían los Gobiernos de izquierda, Paraguay fue suspendido y, posteriormente, al prevalecer la centroderecha, la suspensión de Venezuela fue inevitable. Los enfrentamientos políticos obstaculizan la resolución de problemas que impiden profundizar en la integración económica. Hoy en día existe un Mercosur estancado que no ha cumplido su objetivo inicial: la creación del mercado común. Además, el intervencionismo estatal ha aumentado y la política no solo ha desplazado a la economía, sino que ha producido divisiones entre sus miembros. El organismo regional se ha alejado de lo que Foxley denomina integración productiva. De acuerdo con él, "en casi 60 años de esfuerzos de integración, se han firmado decenas de acuerdos y tratados, pero durante todo ese proceso, la integración desde la base productiva al estilo del Asia o de Europa muestra febles resultados” (2014, p. 17). 
A pesar de los problemas señalados, el Mercosur impulsó el comercio intrazona, aunque a un nivel por debajo del potencial. ${ }^{9}$ Más importante aún, dicho comercio consiste principalmente en bienes manufacturados con mayor valor agregado que el dirigido al resto del mundo, cuyo componente principal son bienes agrícolas. El BID (2013b) señala que las manufacturas de contenido tecnológico medio representan más de la mitad de las ventas intrazona de Argentina y Brasil, y un quinto de las uruguayas. Con los cambios políticos ocurridos en Argentina y Brasil, líderes del Mercosur, es probable que el organismo retome el camino del regionalismo abierto, lo cual facilitaría el proceso de convergencia con la AP.

\section{Alianza del Pacífico: del regionalismo abierto al regionalismo del siglo XXI}

La AP se creó formalmente el 28 de abril de 2011 con la Declaración de Lima, donde los presidentes de Colombia, Chile, México y Perú convinieron en crear un área de integración profunda para alentar el desarrollo económico y social, atraer inversiones, obtener competitividad y proyectarse hacia la región Asia-Pacífico. Posteriormente, en el Acuerdo Marco del 6 de junio de 2012, los países se comprometieron a utilizar la integración regional como instrumento para el desarrollo económico y social que contribuya a resolver los problemas de la pobreza, la exclusión y la desigualdad social. El Acuerdo Marco establece tres objetivos: primero, construir un área de integración profunda donde exista la libre circulación de bienes, servicios, capitales y personas; segundo, impulsar el desarrollo económico y la competitividad; tercero, proyectarse al mundo, en especial, a la zona Asia-Pacífico.

De modo que la AP nace comprometida con el regionalismo abierto, es decir, la creación del espacio comercial preferencial no se hace a expensas del sistema multilateral del comercio y pretende incentivar la competitividad de las empresas locales. La propuesta no es sorprendente, si consideramos que los miembros de la AP han mantenido políticas económicas favorables al mercado desde que implementaron sus programas de ajuste estructural y liberación económica. ${ }^{10}$ De hecho, todos apoyaron la propuesta de los Estados Unidos para crear el Área de Libre Comercio de las Américas (ALCA) (Bernal-Meza, 2015), ${ }^{11}$ y tras su fracaso, firmaron tratados de libre comercio con los Estados Unidos. Briceño (2013) ubica la AP en el eje de la integración abierta, basado en el modelo del Tratado de Libre Comercio de América del Norte.

\footnotetext{
${ }^{9}$ El comercio intrazona ha disminuido y apenas superó $13 \%$ en 2014 y 2015.

${ }^{10}$ Chile lo hizo en la década de 1970, México en 1982 y Colombia y Perú en 1990.

${ }^{11}$ En contraste, los países del Mercosur se opusieron al Área de Libre Comercio de las Américas, principalmente Argentina y Venezuela.
} 
Más importante aún, la AP pretende impulsar la integración profunda, aquella que va más allá de otorgar preferencias arancelarias incorporando normas OMC+ y OMC$\mathrm{X},{ }^{12}$ con el fin de crear el marco regulatorio adecuado para las CGV.

Echebarría y Estevadeordal (2014, p. 32) afirman que los acuerdos de integración profunda, "con disciplinas que van más allá del tema de acceso a mercados, pueden generar más del doble de los encadenamientos productivos que los acuerdos que se enfocan en la eliminación del pago arancelario solamente”. Por otro lado, según la OMC (2011, p. 12), “cuanto más profundo es un acuerdo, mayor es el aumento del comercio en piezas y componentes entre países miembros”. Es decir, mayor es el comercio de CGV, el cual puede traer importantes beneficios para los países en desarrollo. Por ejemplo, la Conferencia de las Naciones Unidas para el Comercio y el Desarrollo (UNCTAD, 2013) encontró una correlación positiva entre la participación en CGV y la tasa de crecimiento del PIB. Además, afirma que a largo plazo las CGV pueden aumentar la capacidad productiva de estos países mediante la diseminación de tecnología y el mejoramiento de la mano de obra.

Las CGV tienen un carácter regional, no global, y forman un patrón del tipo hub-andspoke system, siendo los hubs principalmente los Estados Unidos, Alemania, Japón y China (Baldwin, 2011). Los Estados Unidos y los países con avanzada tecnología están impulsando la gobernanza de las CGV mediante la creación de acuerdos megarregionales que incorporan provisiones OMC+ y OMC-X, como el Acuerdo Transpacífico y la Asociación Transatlántica de Comercio e Inversión. Estos acuerdos buscan armonizar las reglas que regulan la integración profunda, que Baldwin (2011) clasifica en dos grupos: reglas que aseguran el flujo bidireccional de bienes, información, capital y gente; y reglas que garantizan la propiedad de bienes tangibles e intangibles y un buen clima de negocios.

Chile, México y Perú participaron en las negociaciones del Acuerdo Transpacífico, tratado que se firmó el 4 de febrero de 2016 y espera por la ratificación de los Congresos de los países firmantes. Bernal-Meza (2015) afirma que el camino de ingreso de los otros países latinoamericanos en el Acuerdo Transpacífico será por la vía de la AP. Aquellos países en desarrollo que no formen parte de acuerdos megarregionales difícilmente podrán participar en el comercio de CGV, que es el que ha registrado mayor dinamismo en lo que va de siglo. Los estudios de impacto estiman que de concretarse las negociaciones megarregionales las ventas del Mercosur a los Estados Unidos y la Unión Europea podrían caer 7 y 4.9 \%, respectivamente, mientras que las ventas de Brasil a esas regiones caerían entre 5 y 10 \% (Zelicovich, 2016).

${ }^{12} \mathrm{OMC}+$ se refiere a la integración profunda en áreas contempladas por la OMC, por ejemplo, administración aduanera, impuestos a las exportaciones, ayuda estatal y contratación pública. OMC-X alude a la integración profunda en aquellas áreas no cubiertas por acuerdos de la OMC, como derechos de propiedad intelectual, inversión, movimientos de capital, mercado laboral, políticas de competencia y leyes ambientales. 
Los miembros de la AP lucen como los países latinoamericanos mejor posicionados para participar en los acuerdos megarregionales de integración y en las CGV. Además de su compromiso con la integración profunda, son economías abiertas al comercio internacional con cuarenta y seis tratados de libre comercio (TLC) firmados con diferentes países, que incluye la Unión Europea y los Estados Unidos, ${ }^{13}$ principal propulsor del Acuerdo Transpacífico y la Asociación Transatlántica de Comercio e Inversión.

Un objetivo de la AP que merece especial mención es su compromiso para proyectarse hacia el Asia-Pacífico, región que ha registrado gran dinamismo en el comercio internacional y que desempeña un papel cada vez más importante para el comercio de América Latina. En 2015, ya el Asia-Pacífico se había convertido en el destino de 18 \% de las exportaciones de América Latina y el origen de 31 \% de sus importaciones, siendo China el socio más importante (Observatorio América Latina-Asia-Pacífico, 2015).

El comercio intrarregional de la AP es bajo, apenas $3.2 \%$ en 2015, a pesar de que la mayor parte de los aranceles ya habían sido removidos gracias a la firma de TLC entre los países miembros. Durán y Cracau (2016) adelantan dos posibles explicaciones: primero, los miembros de la AP no son socios comerciales naturales; segundo, Colombia, Chile y Perú exportan principalmente materias primas. Sin embargo, esta situación podría cambiar si se saca provecho de la acumulación de origen, contemplada en el Protocolo Comercial firmado en febrero de 2014 y vigente a partir de abril de 2016. La acumulación de origen permite que se incorporen insumos y bienes intermedios de cualquier país de la AP en el bien final, que podrá ser exportado a cualquiera de dichos países. En otras palabras, las materias primas de los cuatro países se consideran como nacionales, lo cual es un elemento fundamental para crear CGV dentro de la región. Además, el Protocolo introduce normas OMC+ y OMC-X sobre contratación pública, inversión y comercio de servicios que facilitan la integración profunda y el comercio de CGV dentro y fuera de la región.

Otros logros de la AP son la creación del Mercado Integrado Latinoamericano, que, al agrupar las bolsas de valores de los países miembros, amplía las fuentes de financiamiento para las empresas de la región y genera nuevas oportunidades de inversión, la eliminación de las visas de negocios y de turismo para los residentes de la AP y la aceptación de 43 países como miembros observadores.

Un hecho que resalta el pragmatismo de la AP es la creación del Consejo Empresarial de la Alianza del Pacífico, cuyos objetivos principales son dar sugerencias para la mejor marcha del proceso de integración, impulsar acciones conjuntas hacia terceros mercados y presentar recomendaciones a las asociaciones empresariales de los cuatro

\footnotetext{
${ }^{13}$ La suscripción de TLC con los Estados Unidos y la Unión Europea por parte de los países centroamericanos, Chile, Colombia, México y Perú ha permitido que estos registren mayores tasas de implementación de medidas de facilitación del comercio en la región (Cepal, 2015), elemento importante para las CGV.
} 
países. De acuerdo con Roberto Paiva, director de Prochile, ${ }^{14}$ la integración "la firman los presidentes, los ministros, pero para que tenga 'carne' la tienen que llevar adelante los empresarios... el gran impulso de la Alianza es haber puesto en un proyecto integracionista a los empresarios por delante" (Custodio, 2016).

\section{Alianza del Pacífico y Mercado Común del Sur: desempeño económico e instituciones}

Tanto el Mercosur como la AP fueron creados con objetivos ajustados al regionalismo abierto. Sin embargo, el Mercosur se alejó del liberalismo económico promoviendo una mayor intervención del Estado en la actividad económica; además, la agenda política cobró especial relevancia y condujo a la confrontación con el poder hegemónico de los Estados Unidos y a enfrentamientos entre los Estados partes. ${ }^{15}$ Los países de la AP, por su parte, mantuvieron sus políticas económicas neoliberales, profundizaron la integración con los Estados Unidos e hicieron de la integración profunda un elemento esencial para formar parte de las CGV. Cabe entonces preguntar: ¿̇cuál de los dos sistemas de integración está mejor preparado para enfrentar los cambios que están ocurriendo en el comercio mundial y convertir la integración regional en un instrumento que ayude a impulsar el desarrollo económico?

Intentaremos responder a la pregunta utilizando diferentes criterios. El primero es la capacidad que tiene cada bloque para enfrentar choques externos. Tomando en cuenta que, a excepción de México, los países que conforman ambos bloques exportan principalmente materias primas, podríamos comparar el desempeño de cada bloque ante la caída en los precios de las materias que se inicia en 2011 y se agudiza a partir de 2014.

En la figura 1, se observa que durante el periodo señalado el PIB disminuyó en los dos bloques, pero en el Mercosur la caída fue más pronunciada y la volatilidad mayor. En cuanto a la inflación, en la AP se mantuvo por debajo de $5 \%$, mientras que en el Mercosur fue de dos dígitos, y a partir de 2013 creció significativamente, principalmente, por los desequilibrios macroeconómicos registrados en Venezuela. Estas dos variables indican que el desempeño económico de los miembros de la AP fue mejor y sus economías mostraron mayor estabilidad ante el shock producido por la caída en los precios de las materias primas. Un resultado importante si consideramos que los estudios empíricos han encontrado una correlación positiva entre la estabilidad económica y el crecimiento (Easterly, 2003).

\footnotetext{
${ }^{14}$ Prochile es la institución gubernamental encargada de promover las exportaciones, la inversión extranjera y el turismo.

${ }^{15}$ Sobre los aspectos políticos de la AP y el Mercosur, ver Bernal-Meza (2015).
} 
Figura 1a. Producto Interno Bruto

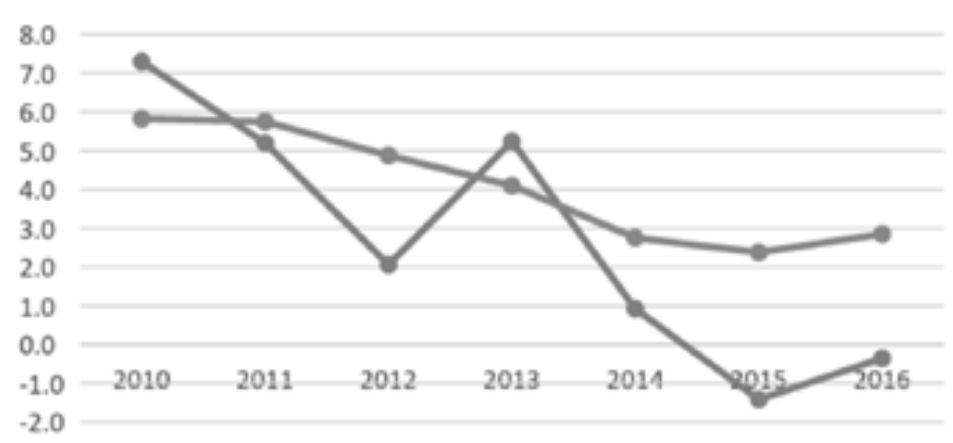

Mercosur AP

Figura 1b. Inflación

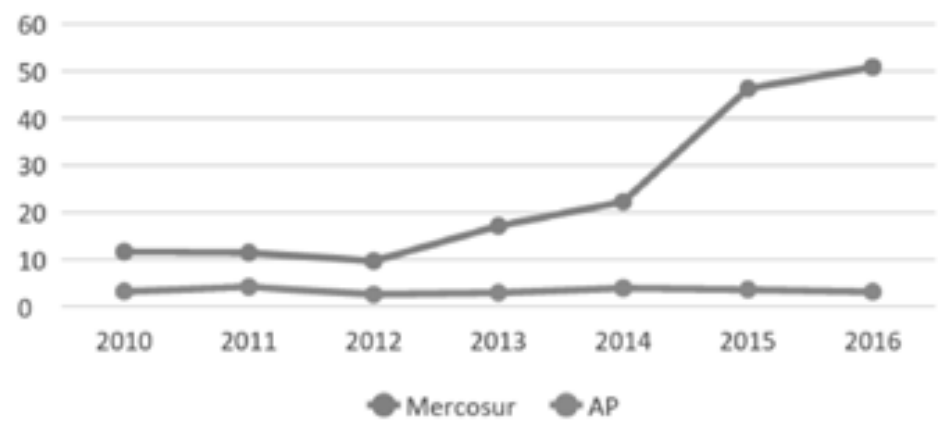

Fuente: FMI

Nota: las cifras de 2016 son estimadas

De acuerdo con el World Economic Forum (2015), la competitividad aumenta la resiliencia de las economías ante los choques externos. La tabla 1 muestra que todos los países de la AP obtuvieron mejores índices de competitividad ${ }^{16}$ que los países pertenecientes al Mercosur, lo cual parece haber incidido en su mejor desempeño macroeconómico.

\footnotetext{
${ }^{16} \mathrm{El}$ índice se basa en 114 indicadores agrupados en 12 pilares: instituciones, infraestructura, ambiente macroeconómico, salud y educación primaria, educación superior y capacitación, eficiencia en el mercado de bienes, eficiencia en el mercado laboral, desarrollo del mercado financiero, facilidad para incorporar tecnología, tamaño del mercado, sofisticación en los negocios e innovación.
} 
Tabla 1. Indice de Competitivdad Global 2015-2016

\begin{tabular}{|l|l|l|}
\hline \multicolumn{1}{|c|}{ Puntuacion } & \multicolumn{1}{c|}{ AP } & \multicolumn{1}{c|}{ Mercosur } \\
\hline 4.58 & Chile & \\
\hline 4.29 & Mexico & \\
\hline 4.28 & Colombia & \\
\hline 4.21 & Peru & \\
\hline 4.09 & & Uruguay \\
\hline 4.08 & & Brasil \\
\hline 3.79 & & Argentina \\
\hline 3.6 & & Paraguay \\
\hline 3.3 & & Venezuela \\
\hline
\end{tabular}

Fuente: World Economic Forum

La competitividad y el comercio internacional están estrechamente relacionados. Cuando un país entra en un acuerdo de integración regional, puede aumentar su competitividad mediante la ampliación de los mercados, que permite alcanzar economías de escala, y la atracción de inversión extranjera, que aporta tecnología y experticia. A su vez, cuanto más competitiva sea la economía, mayor provecho obtendrán los productores locales de la apertura al comercio y la inversión extranjera. En este sentido, los miembros de la AP están mejor posicionados para aprovechar los efectos positivos de la integración regional.

Como señalamos, la integración profunda contempla un conjunto de regulaciones necesarias para la conformación de CGV. Estas buscan garantizar, por un lado, la libre circulación de bienes, capital y personas; por el otro, la propiedad de bienes tangibles e intangibles y un buen clima de negocios. Una manera indirecta de medir la adecuación de las normas de un país para la integración profunda es a través del índice de libertad económica, que incluye variables que miden la protección a la propiedad privada, trabas al comercio internacional y al movimiento de capitales, así como la facilidad de establecer un negocio. En la tabla 2, se puede apreciar que, excepto Uruguay, todos los países del Mercosur están por debajo de los de la AP, por lo cual pone a esta última en mejor posición para avanzar en el comercio de CGV.

Tabla 2. Indice de Libertad Economica 2016

\begin{tabular}{|l|l|l|}
\hline \multicolumn{1}{|c|}{ Puntuacion } & \multicolumn{1}{c|}{ AP } & \multicolumn{1}{c|}{ Mercosur } \\
\hline 77.7 & Chile & \\
\hline 70.8 & Colombia & \\
\hline 68.8 & & Uruguay \\
\hline 67.4 & Peru & \\
\hline 65.2 & Mexico & \\
\hline 61.5 & & Paraguay \\
\hline 56.5 & & Brasil \\
\hline 43.8 & & Argentina \\
\hline 33.7 & & Venezuela \\
\hline
\end{tabular}

Fuente: Heritage Foundation 
Por otro lado, el índice Doing Business del Banco Mundial mide la facilidad para hacer negocios tomando en consideración regulaciones y procesos regulatorios relacionados con el establecimiento y la operación de un negocio. ${ }^{17}$ Nuevamente, todos los países de la AP obtuvieron mejores puntuaciones que los del Mercosur en 2016 (tabla 3).

Tabla 3. Facilidad de Hacer Negocios 2016

\begin{tabular}{|l|l|l|}
\hline \multicolumn{1}{|c|}{ Puntuacion } & \multicolumn{1}{c|}{ AP } & \multicolumn{1}{c|}{ Mercosur } \\
\hline 73.72 & Mexico & \\
\hline 71.49 & Chile & \\
\hline 71.33 & Peru & \\
\hline 70.43 & Colombia & \\
\hline 61.21 & & Uruguay \\
\hline 60.19 & & Paraguay \\
\hline 57.67 & & Brasil \\
\hline 56.78 & & Argentina \\
\hline 35.51 & & Venezuela \\
\hline
\end{tabular}

Fuente: World Bank

De lo anterior se desprende que los miembros de la AP tienen un marco institucional más adecuado que los del Mercosur para implementar las CGV, que han registrado mayor dinamismo en los últimos años y tienen efectos positivos sobre la capacidad productiva y el crecimiento económico de los países en desarrollo.

\section{Alianza del Pacífico y Mercado Común del Sur: ¿convergencia o divergencia?}

El lanzamiento de la AP generó oposición y críticas de algunos países latinoamericanos, principalmente de los miembros de la Alianza Bolivariana para los Pueblos de Nuestra América (ALBA). Durante la cumbre de el ALBA celebrada en julio de 2013, Rafael Correa, presidente de Ecuador, afirmó:

Queremos mucho a Colombia, Perú, Chile, México, pero se enfrentan dos visiones del mundo: el neoliberalismo, el libre comercio, y aquellos que creemos en el socialismo, en garantía de derechos, en zonas libres, pero no para el libre comercio sino libre de hambre, libre de pobreza. (Núñez, 2013)

Tres meses después Evo Morales, presidente de Bolivia, declaró: “Estados Unidos está dividiendo a la Unasur. Los países que conforman la Alianza del Pacífico son parte

\footnotetext{
${ }^{17}$ El índice mide once áreas de regulación: comenzar un negocio, permisos de construcción, obtención de electricidad, registro de propiedad, obtención de crédito, protección de inversionistas minoritarios, pago de impuestos, comercio internacional, cumplimiento de contratos, resolución de insolvencia y regulación del mercado laboral.
} 
de una conspiración que viene desde el norte para dividir y que Unasur no avance hacia la liberación definitiva" (El Tiempo, 2013). Curiosamente, las declaraciones de Nicolás Maduro fueron moderadas y se limitaron a proponer la unión del Mercosur y el ALBA para contrarrestar la influencia de la AP.

En el Mercosur, las reacciones fueron distintas. Paraguay y Uruguay, fuertes críticos de las políticas proteccionistas de Brasil y, especialmente de Argentina, vieron el surgimiento de la AP como una oportunidad. Uruguay, país que había considerado negociar individualmente un TLC con los Estados Unidos, evaluó la posibilidad de hacerse miembro pleno de la AP, pero ingresó como miembro observador en la V Cumbre de la AP celebrada en 2012. ${ }^{18}$ Paraguay lo hizo al año siguiente, tomando en consideración que la AP facilitaría el acceso a los mercados de Asia-Pacífico.

Brasil, líder indiscutible de la región, tomó el surgimiento de la AP de manera pragmática, aceptando la diversidad existente en las estrategias de integración de la región, y su diplomacia, de acuerdo con Goulart (2016), no vio el nuevo sistema de integración como una amenaza al comercio que mantiene con sus países miembros. Brasil tradicionalmente ha mantenido excelentes relaciones con Chile (Fermandois, 2016) y firmó una alianza estratégica con Perú en 2003.

La reacción de Argentina fue diferente. En un principio, Cristina Fernández consideró la AP como "un actor que conspiraba contra la proyección sudamericana del Mercosur" (Bernal-Meza, 2015, p. 14), pero luego cambió su posición y abogó por la aproximación de los dos bloques durante la cumbre del Mercosur de julio de 2013 (Aranda y Riquelme, 2015). De modo que el pragmatismo se impuso sobre la política, lo cual era de esperar por ser Chile el cuarto destino de las exportaciones argentinas.

Los miembros de la AP no querían convertir el organismo en una plataforma para enfrentar el Mercosur. Durante la IV Cumbre de la AP celebrada en junio de 2012 el presidente de Chile, Sebastián Piñera, no descartó la posibilidad de que en el futuro los países del Mercosur quisieran incorporarse a la AP (Diario Libre, 2013). Y ha sido Chile el país que más se ha esforzado por acercar los dos bloques, aprovechando los fuertes vínculos que mantiene con Brasil y Argentina. Uno de los postulados del segundo gobierno de Michelle Bachelet, sobre integración regional, es la idea de la convergencia en la diversidad. La convergencia gradual y pragmática entre la AP y el Mercosur es una tarea que la presidenta considera no solo posible, sino necesaria (Aranda y Riquelme, 2015). En este sentido, el ministro de Relaciones Exteriores, Heraldo Muñoz, afirmó:

No quisiéramos que la Alianza del Pacífico sea concebida como una suerte de bloque político excluyente, que se contraponga al Atlántico. Este no es el bloque de los librecambistas y

${ }^{18}$ Danilo Astori, vicepresidente de Uruguay, lideró el grupo que proponía la membrecía plena. 
eficientes, versus los estatistas ineficientes. Tenemos que tener respeto a los países del Atlántico, y buscar convergencia en la diversidad. (Oppenheimer, 2014)

En la declaración de Punta Mita, con motivo de la IX Cumbre de la AP, se recogió la idea de ampliar el diálogo con los países del Mercosur y últimamente los dos bloques comerciales han explorado las posibilidades para trabajar conjuntamente en la facilitación del comercio. Después de todo "la oposición entre Mercosur y la Alianza del Pacífico es apresurada, intencionada y superficial. Modelos alternativos de integración no implican exclusiones y autonomías" (Turzi, 2014, p. 82).

Los ataques iniciales a la AP, calificándola como un instrumento de los Estados Unidos para enfrentar el ALBA y el Mercosur, perdieron fuerza. Entre otras razones, por el debilitamiento de los Gobiernos de izquierda en la región a partir de la elección de Mauricio Macri en Argentina, la derrota de Evo Morales en el referendo realizado el 21 de febrero de 2016 que impidió su postulación a la presidencia por cuarta vez, los problemas económicos y políticos de Venezuela a causa de la caída de los precios del petróleo y la destitución de Dilma Rousseff. Un factor adicional, que no debe ser subestimado, es el mejor desempeño económico de los miembros de la AP. Estos acontecimientos han favorecido un clima de acercamiento entre la AP y el Mercosur. La integración productiva de estos dos bloques para promover las CGV es necesaria para que América Latina pueda competir en la economía global con productos con mayor valor agregado y así disminuir el peso de los commodities en las exportaciones totales. Según Foxley (2014), este esfuerzo integrador se facilitaría si Brasil asumiera el liderazgo abriendo sus mercados al resto de la región, y los miembros de la AP, por su parte, otorgaran incentivos a las empresas brasileñas para conformar las CGV.

Un obstáculo importante para la integración regional y el establecimiento de las CGV es la infraestructura. Según la Cepal (2015), la inadecuada infraestructura de transporte en América Latina es una de las causas de la poca participación de la región en las CGV. Puertos ineficientes, servicios aéreos poco competitivos y baja calidad o inexistencia de carreteras intrarregionales son algunos de los problemas que fragmentan el mercado regional y dificultan la formación de CGV tanto entre empresas de la región como con empresas de otras regiones. Para atacar estos problemas, se creó la iniciativa del Consejo Suramericano de Infraestructura y Planeamiento de la Unión de Naciones Suramericanas (Unasur), que busca la integración de la infraestructura de los países miembros. Sin embargo, en un informe sobre los avances de la cartera de proyectos de este consejo para 2013, se menciona "la relativa lentitud con que avanza la cartera $(74,1 \%$ de los proyectos se encuentran en etapa de perfil desde el 2010, y de éstos el $30 \%$ desde el 2004)” (BID, 2013a). De modo que el esfuerzo que se está haciendo para desarrollar la infraestructura regional no es suficiente. 
Finalmente, otro problema por resolver es la facilitación del comercio, es decir, la simplificación y armonización de los procedimientos relacionados con el comercio internacional, tales como procedimientos aduanales, de transporte, pagos y seguros (Cepal, 2015). Este tema es importante para las CGV, porque, cuando se divide la producción entre varios países, la cantidad de transacciones fronterizas aumenta. Por otro lado, la facilitación del comercio favorece la internacionalización de las pequeñas y medianas empresas. Es paradójico que para una empresa de la región el costo de negociar con los Estados Unidos sea menor que el costo del comercio intrarregional.

\section{Conclusiones}

La AP y el Mercosur han seguido estrategias de integración regional diferentes. Los países de la AP optaron por una menor intervención del Estado en la economía que favoreció los mercados en busca de eficiencia económica y promovió la integración profunda con el objeto de participar en las CGV, lo cual hizo de la economía el elemento esencial de la integración. Los países del Mercosur, por su parte, favorecieron políticas mercantilistas, desconfiaron de la eficiencia de los mercados y prefirieron la integración hacia dentro; además, le dieron a la política un papel central en el proceso de integración. Los conflictos políticos e ideológicos entre los Estados partes han conducido al Mercosur a una de sus crisis más graves.

Los miembros de la AP registraron mejor desempeño económico durante los últimos cinco años y mayor resiliencia ante la caída en los precios de las materias primas que los del Mercosur. Esto se explica, en gran parte, por sus mejores índices de competitividad. Su mayor competitividad y compromiso con la integración profunda los pone en mejor posición para formar parte de las CGV y convertir la integración regional en motor del crecimiento económico.

A pesar de las diferencias señaladas y de los intentos iniciales de crear rivalidad entre ambos organismos regionales, el futuro de América Latina luce mejor si se logra la convergencia AP-Mercosur y se fomenta la creación de CGV. Para ello, se requiere avanzar en la integración profunda y en la facilitación del comercio. También es fundamental mejorar la infraestructura regional, objetivo que se podría facilitar con el fortalecimiento del Consejo Suramericano de Infraestructura y Planeamiento. En cuanto a la creación de CGV, el liderazgo debería ser asumido por Brasil y México, países que podrían convertirse en hubs apoyados en la robustez de sus sectores industriales.

Con la caída en los precios de las materias primas y el giro hacia la derecha suscitado en el Mercosur, es probable que este organismo vuelva a su objetivo original de hacer del regionalismo abierto un motor del desarrollo económico, lo cual facilitaría la convergencia con la AP. 


\section{Referencias}

Angarita, Y. (23 agosto 2016). Mercosur: cronología de una crisis. En El Universal. Recuperado de http://www.eluniversal.com/noticias/politica/mercosur -cronologia-una-crisis_433046

Aranda Bustamante, G. y Riquelme Rivera, J. (2015). ¿Es posible la convergencia en la diversidad? Chile entre la Alianza del Pacífico y Mercosur. Revista de Relaciones Internacionales, Estrategia y Seguridad, 10(2), 155-178.

Balassa, B. (2014). The theory of economic integration: An introduction. En B. F. Nelsen y A. Stubb (eds.), The European Union: Readings on the theory and practice of european integration ( $4 \cdot^{\mathrm{a}}$ ed., pp. 165-176). Boulder, CO: Lynne Rienner Publishers.

Baldwin, R. E. (2011). 21st Century Regionalism: Filling the Gap between 21st Century Trade and 2oth Century Trade Rules. Recuperado de https://papers.ssrn.com/ sol3/papers.cfm?abstract_id=1869845

Banco Interamericano de Desarrollo (2009). Informe Mercosur n. ${ }^{\circ} 14$. Recuperado de https://publications.iadb.org/handle/11319/2673?locale-attribute=es

Banco Interamericano de Desarrollo (2013a). Carta mensual INTAL n. ${ }^{\circ}$ 207. Recuperado de http://www.oei.es/historico/org57.htm

Banco Interamericano de Desarrollo (2013b). Informe Mercosur n. ${ }^{0} 18$. Recuperado de https://publications.iadb.org/bitstream/handle/11319/6020/Informe \%20 MERCOSUR \%2ON \%C2 \%Bo \%2018 \%20(2012-2013) \%20.pdf?sequence=1

Banco Interamericano de Desarrollo (2015). Informe Mercosur n. ${ }^{\circ}$ 20. Recuperado de https://publications.iadb.org/bitstream/handle/11319/7280/Informe_ MERCOSUR_N_2O_2014_2015_Segundo_Semestre_2014_Primer_Semestre_2015.pdf?sequence $=1$

Bernal-Meza, R. (2015). Alianza del Pacífico versus ALBA y Mercosur: entre el desafío de la convergencia y el riesgo de la fragmentación de Sudamérica. Pesquisa \& Debate, 26(47), 1-34.

Bergamaschine Mata Diz, J. (2005). El sistema de internalización de normas en el Mercosur: la supranacionalidad plena y la vigencia simultánea. Ius et Praxis, 11(2), 227-260.

Briceño Ruiz, J. (2013). Ejes y modelos en la etapa actual de la integración económica regional en América Latina. Estudios Internacionales, 45(175), 9-39. 
Caetano, G. (2011). Breve historia del Mercosur en sus 20 años: coyuntura e instituciones (1991-2011). En G. Caetano (coord.), Mercosur 20 años (pp. 21-71). Montevideo: CEFIR.

Cingano, F. (2014). Trends in income inequality and its impact on economic growth. OECD Social, Employment and Migration Working Papers, 163.

Comisión Económica para América Latina y el Caribe (Cepal) (1994). Open regionalism in Latin America and the Caribbean: Economic integration as a contribution to changing productions patterns with social equity. Santiago de Chile: Naciones Unidas.

Comisión Económica para América Latina y el Caribe (Cepal) (2015). Panorama de la inserción internacional de América Latina y el Caribe. La crisis del comercio regional: diagnóstico y perspectivas. Santiago de Chile: Naciones Unidas.

Conferencia de las Naciones Unidas para el Comercio y el Desarrollo (UNCTAD) (2013). World investment report 2013: Global value chains: Investment and trade for development. Nueva York: UNCTAD.

Custodio, L. (8 febrero 2016). Alianza del Pacífico entra en vigencia en el mes de abril. En Elpais.com.uy. Recuperado de http://www.elpais.com.uy/economia-y-mercado/ alianza-pacifico-entra-vigencia-abril.html

Diario Libre (10 junio 2013). La Alianza acelera la integración política y económica de sus cuatro socios. Recuperado de https://www.diariolibre.com/economia/ economia-personal/alianza-del-pacfico-acelera-la-integracin-poltica-y-econmica-de-sus-cuatro-socios-PDDL387585

Doing Business (2015). Doing Business 2016: midiendo la calidad y eficiencia regulatoria. Washington, D. C.: Banco Mundial.

Durán Lima, J. E. y Cracau, D. (2016). The Pacific Alliance and its economic impact on regional trade and investment Evaluation and perspectives. Santiago de Chile: Naciones Unidas.

Easterly, W. (2003). En busca del crecimiento: andanzas y tribulaciones de los economistas del desarrollo. Barcelona: Bosch.

Echebarría, L. E. y Estevadeordal, A. (2014). Alianza del Pacífico: un nuevo motor de integración regional. En A. Foxley y P. Meller (eds.), Alianza del Pacífico: en el proceso de integración latinoamericana (pp. 27-42). Santiago de Chile: Cieplan.

EFE (3 agosto 2016). Maduro dice que presidirá "plenamente" el Mercosur y reta a los socios del bloque. Recuperado de http://www.efe.com/efe/america/politica/ 
maduro-dice-que-presidira-plenamente-el-mercosur-y-reta-a-los-socios-delbloque/20000035-3003303

El Nacional (31 julio 2016). Mauricio Macri: "En Venezuela hay una violación absoluta de los derechos humanos”. Recuperado de http://www.el-nacional.com/noticias/ historico/mauricio-macri-venezuela-hay-una-violacion-absoluta-los-derechoshumanos_21951

El Tiempo (15 octubre 2013). Evo arremete contra la Alianza del Pacífico. Recuperado de http://www.eltiempo.com/archivo/documento/CMS-13123127

European Comission (s. f.). Regional Integration. Recuperado de http://ec.europa.eu/ europeaid/sectors/economic-growth/regional-integration_en

Fermandois, J. (2016). Brazil: Chile's mythical ally. En Gian Luca, G. y M. H. Tavares de Almeida (eds.), Foreign policy responses to the rise of Brazil: Balancing power in emerging states (pp. 77-89). Londres: Palgrave Macmillan.

Foxley, A. (2014). Nuevo desafío para América Latina: integración productiva. En A. Foxley y P. Meller (eds.), Alianza del Pacífico: en el proceso de integración latinoamericana (pp. 13-26). Santiago de Chile: Cieplan.

Gil, L. y Paikin, D. (2013). Mapa de la integración en América Latina: procesos e instituciones. Nueva Sociedad. Recuperado de http://www.ba.unibo.it/risorse/files/ GilInt.RegionalenAL.pdf

Goulart Menezes, R. (2016). Brasil y la integración suramericana en el siglo XXI: impases y desafíos. En M. I. Matus Matus y G. Aranda Bustamante (eds.), A 100 años del ABC: desafíos y proyecciones en el marco de la integración regional (pp. 133-148). Santiago de Chile.

Heinonen, H. (2006). Regional integration and the state: The changing nature of sovereignty in Southern Africa and Europe (Tesis de doctorado, University of Helsinki, Helsinki, Finlandia).

Inter-American Development Bank (2012). Shaping the Future of the Asia and the Pacific-Latin America and the Caribbean Relationship. Washington D. C.

Makuc, A., Duhalde, G. y Rozemberg, R. (2015). La negociación Mercosur-Unión Europea a veinte años del Acuerdo Marco de Cooperación: ¿̇quo vadis? Washington D. C.: Banco Interamericano de Desarrollo.

Martínez Castillo, A. (2015). De la Comunidad Andina de Naciones al Mercado Común del Sur: nueva estrategia de integración de Venezuela. Colombia Internacional, $83,203-233$. 
Noticia al Día (12 julio 2016). Paraguay: No queremos que presidencia del Mercosur sea instrumento de conflictos. Recuperado de http://noticiaaldia.com/2016/o7/paraguay-no-queremos-que-presidencia-de-mercosur-sea-instrumento-de-conflictos/

Núñez, R. (4 agosto 2013). El ALBA declara la guerra a la Alianza del Pacífico. En Infolatam. Recuperado de http://www.infolatam.com/2013/o8/o4/ el-alba-declara-la-guerra-a-la-alianza-del-pacifico/

Observatorio América Latina-Asia-Pacífico (2015). Boletín Estadístico América LatinaAsia-Pacífico, 8.

Oppenheimer, A. (17 mayo 2014). El futuro de la Alianza del Pacífico. En eju! Recuperado de http://eju.tv/2014/o5/el-futuro-de-la-alianza-del-pacfico/

Organización Mundial del Comercio (OMC) (2011). Informe sobre el comercio mundial 2011. Ginebra: OMC

Picketty, T. (2014), Capital in the XXI century. Cambridge: Harvard University Press.

Riggirozzi, P. y Tussie, D. (2012). The rise of poshegemonic regionalism in Latin America. En P. Riggirozzi y D. Tussie (eds.), The rise of poshegemonic regionalism: The case of Latin America (pp. 1-16). Nueva York: Springer.

Rivas Molina, F. (2 abril 2017). Mercosur aplica la cláusula democrática a Venezuela. En El País. Recuperado de http://internacional.elpais.com/internacional/2017/o4/o1/america/1491076022_326137.html

Sanahuja Perales, J. A. (2007). Regionalismo e integración en América Latina: balance y perspectivas. Pensamiento Iberoamericano, 75-106.

Sanahuja Perales, J. A. (2012). Regionalismo posliberal y multilateralismo en Sudamérica: el caso de Unasur. En A. Serbin, L. Martínez y H. Ramanzini Júnior (coords.), El regionalismo "posliberal" en América Latina y el Caribe: nuevos actores, nuevos temas, nuevos desafios (pp. 19-71). Buenos Aires: Coordinadora Regional de Investigaciones Económicas y Sociales.

Tremolada Álvarez, E. (2014). ¿La Alianza del Pacífico facilita la inserción de Colombia en la región Asia-Pacífico? Papel Político, 19(2), 721-752.

Tugores, J. (2006). Integración regional y políticas públicas: evaluación de la experiencia europea y posibles implicaciones para la integración latinoamericana. México: Naciones Unidas.

Turzi, M. (2014). Asia y la ¿(des) integración latinoamericana? Nueva Sociedad, 250, 78-87.

World Economic Forum (2015). The Global Competitiveness Report 2015-2016. Recuperado de http://reports.weforum.org/global-competitiveness-report-2015-2016/ 
Zelicovich, J. (2015). El Mercosur a 20 años del Protocolo de Ouro Preto: un blance de la dimensión comercial. Revista Latinoamericana de Desarrollo Económico, 24, 97-120.

Zelicovich, J. (2016). El Mercosur frente al "regionalismo del siglo XXI": algunas claves para la comprensión del devenir del proceso de integración. Revista Aportes para la Integración Latinoamericana, 22(34), 1-27. Recuperado de http://sedici.unlp. edu.ar/handle/10915/53785 